\title{
Ion wave breaking acceleration
}

\author{
B. Liu, ${ }^{1,2,3,{ }^{*}}$ J. Meyer-ter-Vehn, ${ }^{4}$ K-U. Bamberg, ${ }^{1}$ W. J. Ma, ${ }^{2}$ J. Liu, ${ }^{2,3}$ X. T. He, ${ }^{2,3}$ \\ X. Q. Yan, ${ }^{2}$ and H. Ruhl ${ }^{1, \dagger}$ \\ ${ }^{1}$ Institute for Computational and Plasma Physics, Ludwig-Maximilian-Universitaet, \\ 80333 Muenchen, Germany \\ ${ }^{2}$ Key Laboratory of HEDP of the Ministry of Education, CAPT, \\ and State Key Laboratory of Nuclear Physics and Technology, Peking University, Beijing 100871, China \\ ${ }^{3}$ Institute of Applied Physics and Computational Mathematics, Beijing 100088, China \\ ${ }^{4}$ Max-Planck-Institut für Quantenoptik, D-85748 Garching, Germany
}

(Received 27 January 2016; published 5 July 2016)

Laser driven ion wave breaking acceleration (IWBA) in plasma wakefields is investigated by means of a one-dimensional (1D) model and 1D/3D particle-in-cell (PIC) simulations. IWBA operates in relativistic transparent plasma for laser intensities in the range of $10^{20}-10^{23} \mathrm{~W} / \mathrm{cm}^{2}$. The threshold for IWBA is identified in the plane of plasma density and laser amplitude. In the region just beyond the threshold, self-injection takes place only for a fraction of ions and in a limited time period. This leads to well collimated ion pulses with peaked energy spectra, in particular for 3D geometry.

DOI: 10.1103/PhysRevAccelBeams.19.073401

With the advent of high-power lasers with focused intensities up to $10^{22} \mathrm{~W} / \mathrm{cm}^{2}$, the generation of laserdriven ion beams has found much attention. The laser beams drive relativistic electrons, separating them from the ions and producing electrostatic fields up to $\mathrm{TV} / \mathrm{cm}$. Ion motion then follows on a longer time scale due to the large ion mass. Various target configurations have been considered, and the reader may refer to a recent review [1] of laser-driven ion acceleration for more information. These beams may become important, e.g. for tumor therapy as a most prominent application [2-4].

A laser pulse propagating in plasma produces a wake, a region of perturbed electron and ion density downstream from the pulse. Self-injection of electrons in laser wakefield acceleration [5-7] is a major mechanism for generating electron beams. Here we extend the scheme to selfinjection of ions. The present scheme is related to socalled hole-boring (HB) acceleration, where electrons are driven into a semi-infinite overcritical plasma, and the electrostatic field then drags all ions continuously forming a double layer wakefield [8-11]. The new observation reported here is that, in plasma at near-critical density and still relativistically transparent, the evolution is more gentle, and only a fraction of ions is injected into the wake over a limited time period. This leads to superior ion pulses with peaked energy spectra; in particular in realistic

\footnotetext{
*binliu271@gmail.com

hartmut.ruhl@physik.uni-muenchen.de
}

Published by the American Physical Society under the terms of the Creative Commons Attribution 3.0 License. Further distribution of this work must maintain attribution to the author $(s)$ and the published article's title, journal citation, and DOI. three-dimensional (3D) geometry, the injection occurs localized close to the laser axis producing highly directed bunches. The alternative scheme of light sail acceleration [12-14], in which ultrathin foils are driven by light pressure, is sensitive to Rayleigh-Taylor instability [15] that tends to degrade beam quality; a detailed 3D comparison with our work still needs to be done. Generically, the new regime may be also viewed as emerging from an electron bubble wakefield that accumulates ions in the center. When driven strongly, this comoving ion wave breaks, and self-injection of background ions occurs which are then accelerated in the front part of the wake. This is similar to ion acceleration in the bubble regime [16-18]; since ion self-injection tends to destroy the downstream part of the bubble, a plasma of two ion species was used in Refs. $[16,18]$, where all lighter ions were trapped and the heavier ones formed a stabilizing background. For the present work, a plasma with a single ion component (protons) is chosen. The bubble then changes into a double layer wake configuration; we call the regime ion wave breaking acceleration (IWBA).

In Fig. 1, we consider 1D particle-in-cell (PIC) simulations, using the code PSC [19], and a corresponding 1D model is sketched in Fig. 2. The simulations use a constant laser pulse with intensity $I_{L}=5 \times 10^{21} \mathrm{~W} / \mathrm{cm}^{2}\left(a_{0}=44\right.$ for wavelength $1 \mu \mathrm{m}$ ) of semi-infinite duration, a steep front edge rising over one laser period, and circular polarization. Here $a_{0}=e E_{L} / m_{e} c \omega_{L}, e$ and $m_{e}$ denote electron charge and mass, respectively, $c$ is the vacuum light velocity, $E_{L}$ the laser electric field, and $\omega_{L}$ the laser frequency.

Figure 1(a) refers to a hydrogen plasma with initial density of $n_{0}=0.2 n_{c}$, and shows a snapshot of the plasma evolution at time 243 fs. Here $n_{c}=m_{e} \omega_{L}^{2} \epsilon_{0} / e^{2}$ is the 
(a) $n_{0}=0.2 n_{c}$ at $243 \mathrm{fs}$

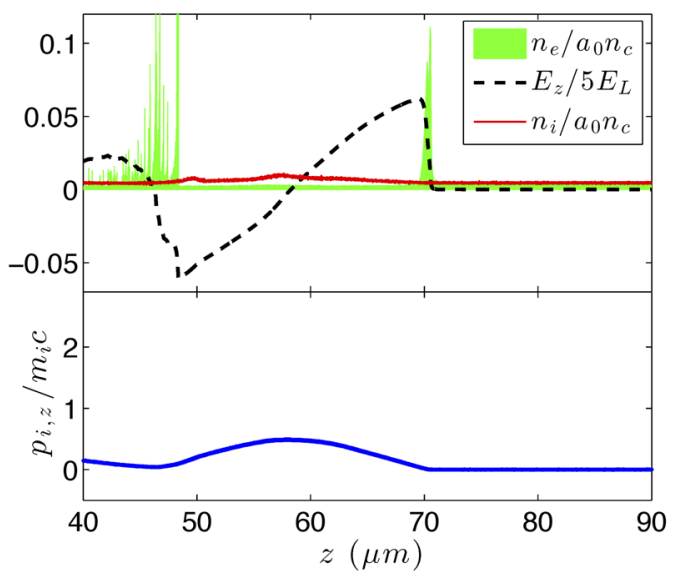

(c) $n_{0}=2.8 n_{c}$ at $333 \mathrm{fs}$

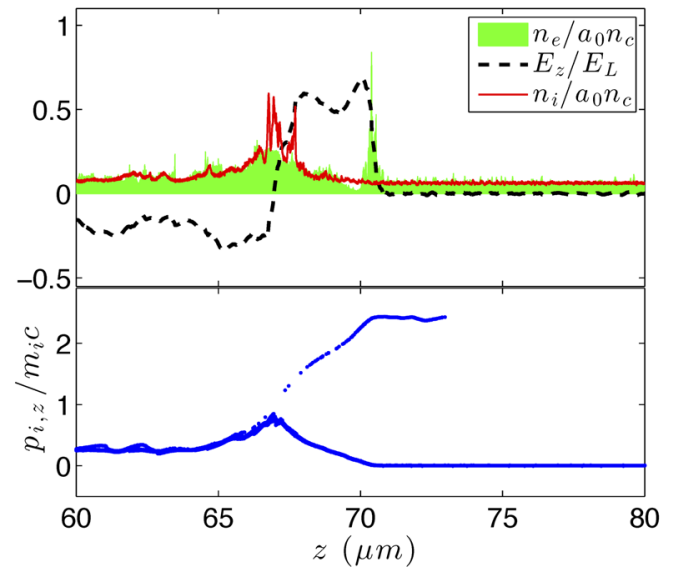

(b) $n_{0}=2.8 n_{c}$ at $93 \mathrm{fs}$

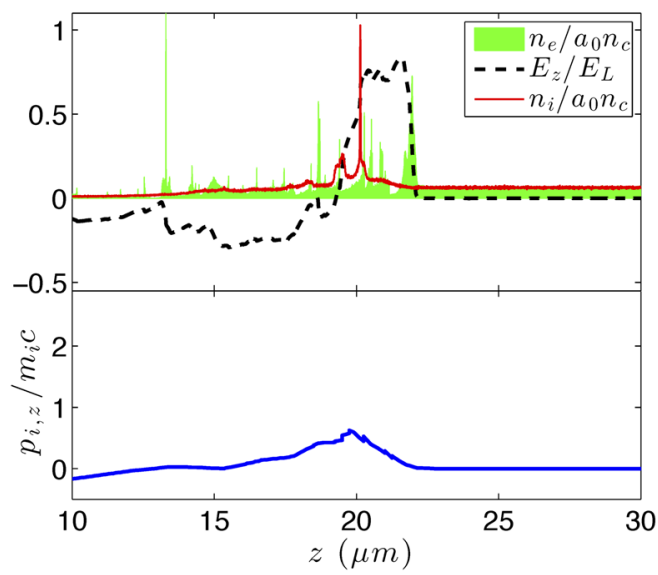

(d) Ion spectrum

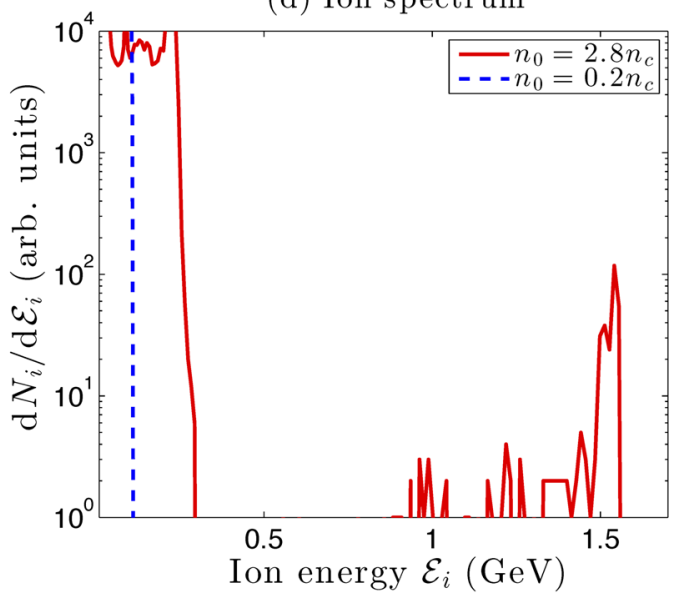

FIG. 1. Results of 1D-PIC simulations. A laser pulse of amplitude $a_{0}=44$ (not shown) is incident from the left, driving a wakefield. Electron and ion densities as well as electric field are plotted for two different initial plasma densities: (a) $0.2 n_{c}$, (b) and (c) $2.8 n_{c}$; lower parts show ion phase space in blue. Results in (b) refer to an early time (93 fs), when the ion wave is just before breaking, while those in (c) refer to a later time (333 fs), when the ion wave is broken and accelerated ions have overtaken the electron layer at the laser front; (a) corresponds to a linear ion wave developing at low plasma density; (d) ion energy spectra corresponding to (c) and (a).

critical density of plasma, $\epsilon_{0}$ is the vacuum permittivity. The plasma is initially distributed uniformly from $z=$ $10 \mu \mathrm{m}$ to $z=300 \mu \mathrm{m}$ with temperature $3 \mathrm{keV}$. The simulation cell size is $5 \mathrm{~nm}$, and 100 particles per species per cell are used. The laser pulse (not shown) drives an electron layer in $z$-direction. It generates the typical electron wake with a sawtooth-shaped electric field. This configuration is modeled in Fig. 2(a) with maximum electrostatic field $E_{\max }$ at the laser front position $z_{A}$ and extending from $z_{A}$ to $z_{B}$. The wake moves with phase velocity $v_{\mathrm{ph}}$. Ions form essentially a uniform stationary background. However, the wakefield drags also the ions exciting a linear ion wave with a density peak in the wake's center. In a frame comoving with the wake (denoted by primed quantities in Fig. 2, lower part), ions enter the wake at velocity $-v_{\mathrm{ph}}$, slow down to $v_{C}{ }^{\prime}$ in the middle, where $E_{z}$ vanishes, and then accelerate again. This holds for not too high laser drive or low enough target density.
Driving the wake more strongly, the ion wave develops a high density crest [see Fig. 2(b), upper part] and eventually breaks [Fig. 2(c)]. This is the point when the ion velocity approaches the wake velocity and overtakes it such that ions are captured by the positive branch of the wakefield and are further accelerated along the dashed trajectory in Fig. 2(c). When catching up with the front and leaving the wake at $z_{A}$, according to relativistic velocity addition, the final ion kinetic energy in the lab frame is given by

$$
\mathcal{E}_{i, \text { peak }}=2 \gamma_{\mathrm{ph}}^{2} \beta_{\mathrm{ph}}^{2} m_{i} c^{2}
$$

here $\gamma_{\mathrm{ph}}=1 / \sqrt{1-\beta_{\mathrm{ph}}^{2}}, \beta_{\mathrm{ph}}=v_{\mathrm{ph}} / c$, and $m_{i}$ denotes ion mass. This stage is observed in Figs. 1(b) and 1(c), corresponding to a simulation at different times $93 \mathrm{fs}$ and 333 fs with same laser amplitude $a_{0}=44$, yet density increased to $2.8 n_{c}$. Branches of ions, captured and 


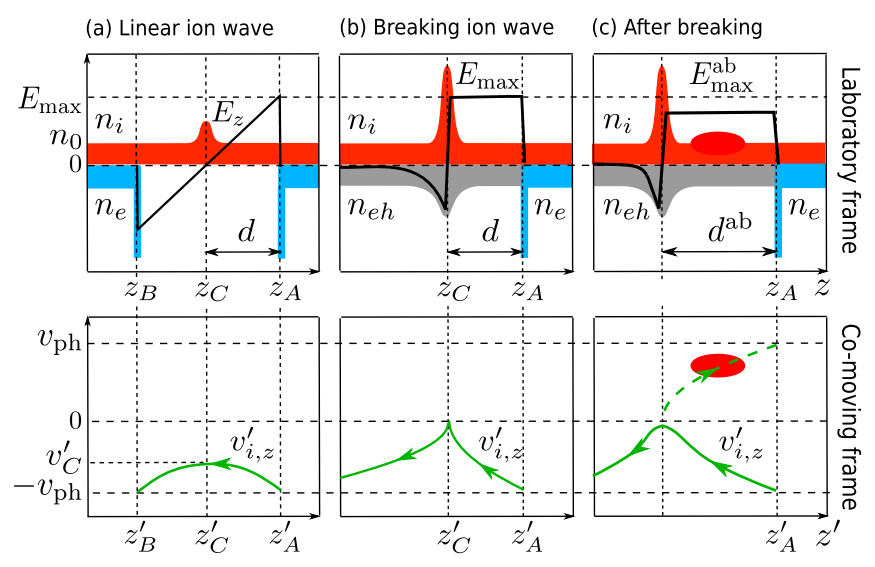

FIG. 2. Schematic figure of ion dynamics, modeling the results of Fig. 1. Density distribution of ions $\left(n_{i}\right)$, cold electrons $\left(n_{e}\right)$, and heated electrons $\left(n_{e h}\right)$ are denoted by red, blue, and gray areas, respectively. The charge separation electric field $E_{z}$ along the propagation direction is denoted by black solid lines. The lower panels show trajectories (green lines) in ion phase space $\left(z, v_{i, z}\right)$ in a frame (marked by apostrophes) comoving with the wake at phase velocity $v_{\text {ph }}$. Column (a) refers to ion waves with small amplitude, where ions just pass through, while (b) refers to a breaking ion wave where ion velocity just reaches $v_{\mathrm{ph}}$, and (c) refers to a stage after breaking when background ions have been partially injected and are accelerated to the right (indicated by a red spot). The wakefield is then more expanded and weaker such that further injection is suppressed. In cases (b) and (c), the downstream cold electron layer has disappeared, and a charge double layer wakefield has formed between the laser-driven front electrons and the trailing ions; it is modeled by width $d$ and constant field $E_{\max }$ in (b), and $d^{\mathrm{ab}}$ and $E_{\max }^{\mathrm{ab}}$ in (c).

accelerated in the wakefield, now show up conspicuously in the phase plot of Fig. 1(c) (lower panel). The corresponding ion energy spectrum is depicted in Fig. 1(d). A high-energy peak separated from the low-energy background ions appears at $1.5 \mathrm{GeV}$. This energy agrees well with Eq. (1) when taking the phase velocity $\beta_{\mathrm{ph}} \sim 0.67$ from the simulation.

At ion wave breaking, the wake configuration changes significantly; it is modeled in Figs. 2(b) and 2(c) (upper panel). The electron void has filled with energetic electrons, in particular downstream from the ion crest. Only the ion layer and the electron layer at the laser front survive with an almost constant electric field in between. This square-wave wake configuration is sensitive to ion injection. At threshold referred to Fig. 1(b), the wake's field is $0.8 E_{L}$ and extends over $2 \mu \mathrm{m}$. After injection in Fig. 1(c), one finds $0.5 E_{L}$ and $3 \mu \mathrm{m}$, and there is no further injection. This is an important feature in the region just beyond the IWBA threshold; it leads to the peaked energy spectrum [see Figs. 1(d) and 5(a)].

In Fig. 3(a) we show peak ion energy (below IWBA threshold it is maximum ion energy) in the $a_{0}, n_{0} / n_{c}$ parameter plane, as obtained from 1D-PIC simulation. (a) $\mathcal{E}_{i, \text { peak }}(\mathrm{GeV})$

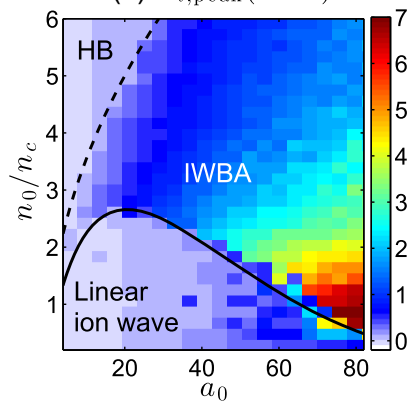

(c) Laser velocity

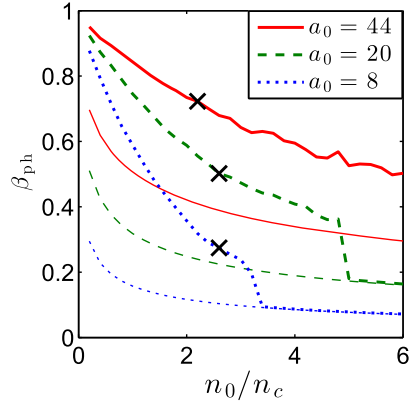

(b) Threshold ion energy

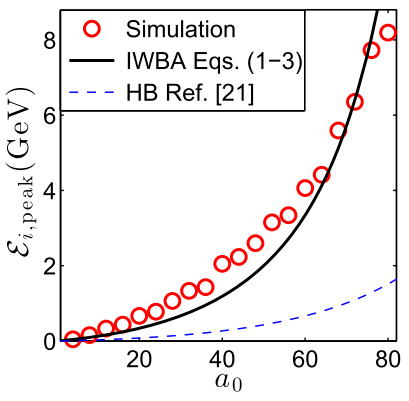

(d) Peak ion energy

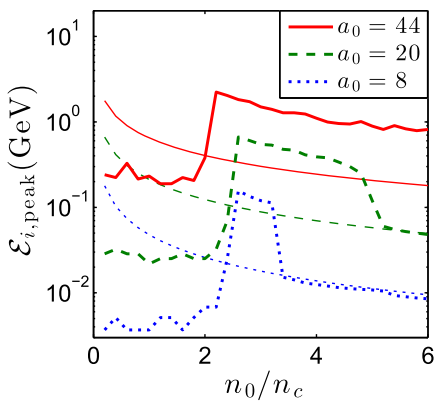

FIG. 3. 1D model and simulation results. (a) Peak ion energy $\mathcal{E}_{i \text {,peak }}$ (color scale) obtained from simulations plotted in plane of laser amplitude $a_{0}$ and density $n_{0} / n_{c}$. The black solid line shows the onset of IWBA derived from Eqs. (2)-(3), the black dashed line denotes the border to hole-boring (HB) (full ion trapping) as obtained from simulations. (b) Maximum of peak ion energy $\mathcal{E}_{i \text {,peak }}$ versus $a_{0}$; red circles from simulations, black solid line from Eqs. (1)-(3), blue thin dashed line from HB model [21]. (c) Laser propagation velocity $\beta_{\text {ph }}$ and (d) peak ion energy $\mathcal{E}_{i \text {,peak }}$ as a function of density $n_{0} / n_{c}$ for $a_{0}=44$ (red thick solid line), $a_{0}=20$ (green thick broken line), and $a_{0}=8$ (blue thick dotted line) obtained from simulation. The corresponding thin lines show the HB model. The black crosses in (c) denote $\beta_{\mathrm{ph}}$ at IWBA threshold as obtained from simulations.

IWBA appears as a separate regime, different from HB. The onset of IWBA is marked by the black solid curve. It is obtained from an analytical model combining the equation for energy conservation in the comoving frame

$$
q_{i} E_{\mathrm{max}} \gamma_{\mathrm{ph}} d=\left(\gamma_{\mathrm{ph}}-1\right) m_{i} c^{2}
$$

where $q_{i}$ denotes ion charge, and the equation expressing balance of laser pressure and electrostatic pressure at the laser front

$$
\frac{1-\beta_{\mathrm{ph}}}{1+\beta_{\mathrm{ph}}} \frac{2 I}{c}=\frac{1}{2} \epsilon_{0} E_{\mathrm{max}}^{2}
$$

These model equations are closed by using estimates for $E_{\max }=\kappa e n_{0} d / \epsilon_{0}$ and $d=\left(1+\beta_{\mathrm{ph}}\right) c /\left(\omega_{L} \sqrt{n_{0} /\left(\delta a_{0} n_{c}\right)}\right)$, where $\kappa$ and $\delta$ are free parameters. Adjusting them to 1D simulations, we find $\kappa \approx 1.8$ and $\delta \approx 2.1$. The black solid 
lines in Figs. 3(a,b) correspond to the model. It reproduces the onset of the IWBA region in the $\left(a_{0}, n_{0} / n_{c}\right)$ plane quite well. In the IWBA region the plasma is relativistically transparent. It becomes opaque at the dashed black line, marking the borderline to the HB regime. The transition from relativistic transparent to opaque plasma has been studied in [20]; here we find that the dashed line is well fitted by $n_{0} / n_{c} \approx \sqrt{a_{0} / 0.8}$.

Let us recall that $\mathrm{HB}$ scaling is derived from an equation similar to Eq. (3) [21], where incident laser momentum flux [1.h.s. of Eq. (3)] is balanced by the total ion momentum flux $2 n_{0} \gamma_{\mathrm{ph}}^{2} \beta_{\mathrm{ph}}^{2} m_{i} c^{2}$ [21]. This already determines the wake velocity $\beta_{\mathrm{ph}}$ as a function of $a_{0}$ and $n_{0} / n_{c}$. In Figs. 3(b)-3(d), results of the full 1D simulations are compared with HB scaling results (thin lines). In Fig. 3(c) it (a) $84 \mathrm{fs}$

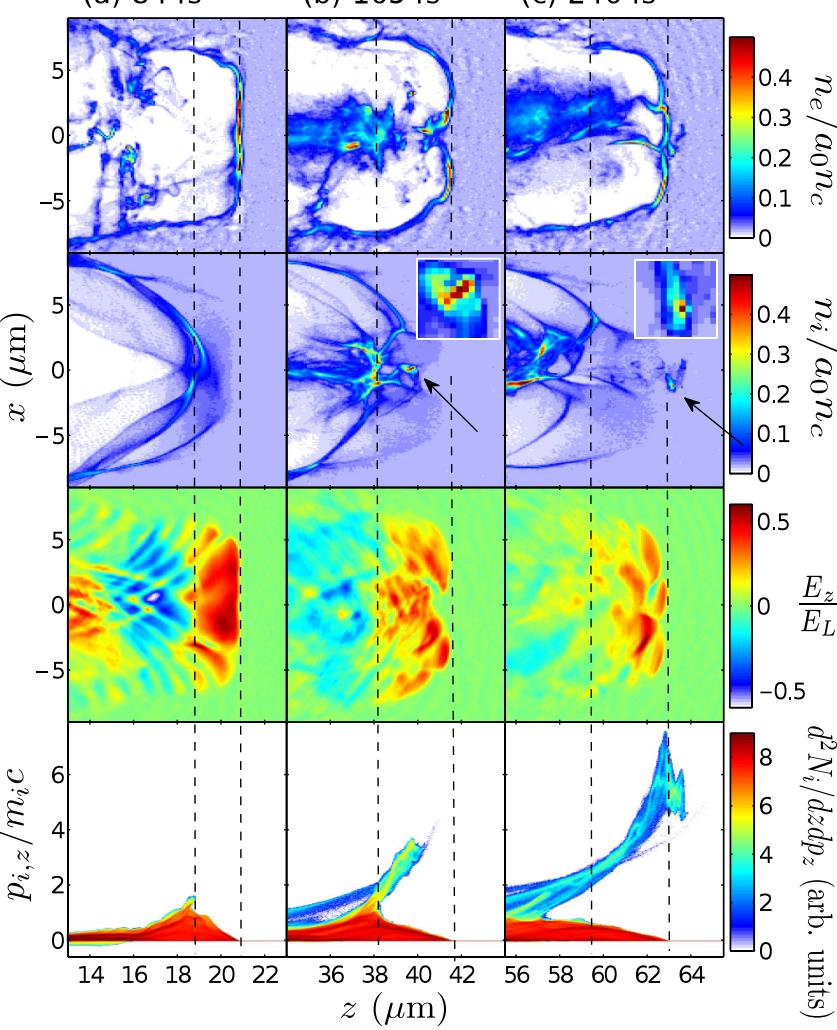

FIG. 4. Results of a 3D-PIC simulation with initial plasma density $n_{0}=3 n_{c}$ and laser amplitude $a_{0}=155$; results are shown as cuts in $(x, z)$ plane at $y=-0.1 \mu \mathrm{m}$. From left to right, results at different times: (a) $84 \mathrm{fs}$, (b) $165 \mathrm{fs}$, (c) $246 \mathrm{fs}$. From top to bottom, distribution of electron density $\left(n_{e} / a_{0} n_{c}\right)$, ion density $\left(n_{i} / a_{0} n_{c}\right)$, electric field $E_{z} / E_{L}$, and ions in $\left(z, p_{z}\right)$ phase space (arbitrary units). The dashed black vertical lines mark the location of the laser-driven electron layer (right line) and the trailing ion layer (left line) with the positive electric field in between. Inserts $(1 \mu \mathrm{m} \times 1 \mu \mathrm{m})$ in the ion density panels show blow-ups of the ion blobs (marked by black arrows), captured and accelerated in this field [column (b)] and just emerging in front of the electron layer [column (c)]. is seen that $\beta_{\mathrm{ph}}$ coincides with the HB scaling for opaque plasma at sufficiently large density, but jumps up when the plasma becomes transparent. This leads to peak ion energies in IWBA regime almost one magnitude larger than the HB prediction [see Figs. 3(d) and (b)].

In Figs. 4 and 5, we show results of full-scale 3D PIC simulations. 3D simulations are numerically quite demanding and produce very complex plasma dynamics, depending significantly on initial parameters. Nevertheless, onset of IWBA and peak ion energies are well described by the $1 \mathrm{D}$ model except that one obtains $\kappa \approx 3.5$ and $\delta \approx 0.5$ by fitting to 3D simulations. In this paper, we have picked one illustrative reference case and have then explored the dependence on density and laser intensity.

A strong laser pulse is chosen for the reference case with Gaussian transverse profile and super-Gaussian in time: $a(r, t)=a_{0} \exp \left(-\left(r / r_{0}\right)^{2}\right) \exp \left(-\left(\left(t-t_{0}\right) / \tau\right)^{2 l}\right)$, here $a_{0}=155, \quad r_{0}=6 \mu \mathrm{m}, \quad t_{0}=20 \mathrm{fs}, \quad \tau=16.7 \mathrm{fs}, \quad$ and $l=15$. The leading edge rises from $a=0.01 a_{0}$ to $a=0.99 a_{0}$ over one laser cycle. The laser pulse is circularly polarized and has a wavelength of $1 \mu \mathrm{m}$. It propagates along the $z$-direction. It is incident on hydrogen plasma extending from $z=5 \mu \mathrm{m}$ to $z=80 \mu \mathrm{m}$ with initial uniform density of $n_{0}=3 n_{c}$ and temperature of $87 \mathrm{keV}$. The simulation box is $80 \times 32 \times 32 \mu \mathrm{m}^{3}(Z X Y)$, with $1000 \times 400 \times 400$ cells, and 8 particles per species per cell are used.

Figure 4 shows the distributions of electron and ion density, longitudinal electric field $E_{z}$, and ion distribution in phase space $\left(z, p_{z}\right)$ for three different times. The vertical dashed lines mark the location of the electron layer and the trailing ion layer. At a time of $84 \mathrm{fs}$, the wake is well developed $\left(E_{\max } \sim 0.4 E_{L}\right.$ and $\left.d \sim 2 \mu \mathrm{m}\right)$, and the ion momentum distribution peaks at $1.6 m_{i} c$. It corresponds
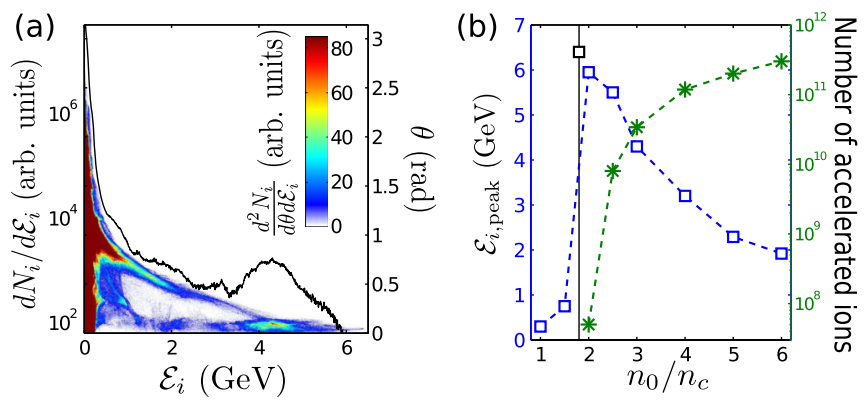

FIG. 5. 3D-PIC simulation results. (a) Ion energy spectrum (black line) and angular-spectrum distribution (color scale) at time 246 fs for the simulation displayed in Fig. 4. (b) Peak ion energy $\mathcal{E}_{i \text {,peak }}$ (blue squares) and numbers of accelerated ions (green asterisks) for different initial plasma densities and laser amplitude $a_{0}=155$, same as in Fig. 4. The black solid vertical line and black square mark the threshold density and the corresponding ion energy as obtained from Eqs. (1)-(3). For $1 n_{c}$ and $1.5 n_{c}$, the maximum energies are plotted since there are no spectral peaks. 
to an ion velocity of $0.85 c$ just approaching the wake velocity of $\sim 0.86 c$ at which ion wave breaking sets in. This is then seen in column (b) at $165 \mathrm{fs}$ with trapped and accelerated ions now clearly sticking out in momentum phase space.

At this time, the ion layer is strongly broken. Most conspicuously, however, an ion bullet (marked by the black arrows and shown as the blow-up in the insert) has separated from the center of the ion layer and is accelerating in the wakefield. The strength of the field reduces to about $0.2 E_{L}$. and the distance between the two charged layers expands to about $3.5 \mu \mathrm{m}$. The bullet is in a volume of about $(0.5 \mu \mathrm{m})^{3}$ and contains about $10^{10}$ ions with energies $>3.5 \mathrm{GeV}$. This corresponds to a bullet density in the order of $10^{23} \mathrm{~cm}^{-3}$. In the final ion spectrum, shown in Figs. 5(a), these ions form the spectral peak around $4.3 \mathrm{GeV}$. The bullet moves under a slight angle to the laser direction with an angular spread of $60 \mathrm{mrad}$; this amounts to an emittance of $10^{-2} \mathrm{~mm}$ mrad. It is also remarkable that the bullet is seen in Fig. 4(c) in the electron density, but not in the electric field plot. This suggests that the bullet is quasineutral and is not subject to Coulomb explosion. Indeed, the bullet shows no expansion after leaving the electron layer in column (c) of Fig. 4. In Fig. 5(b), peak ion energy and number of accelerated ions, obtained from 3D simulations, are shown for different densities and $a_{0}=155$. IWBA sets in at a density somewhat below $n_{0}=2 n_{c}$. From Eqs. (1), (2), (3), the theoretical model predicts $\mathcal{E}_{i \text {,peak }}=6.4 \mathrm{GeV}$ at a threshold density $n_{0}=1.8 n_{c}$; they are shown as a black square and a black vertical line in Fig. 5(b), respectively.

We have also performed 3D simulations for lower laser amplitudes $a_{0}$ and have obtained similar results. For example, a $1 \mathrm{GeV}$ quasi-monoenergetic proton beam is found with $a_{0}=80$ and $n_{0}=8 n_{c}$. However, some general observations should be noted here. Since the tendency for destruction of the electron layer due to Rayleigh-Taylor-like instability increases with ion injection, the window of initial plasma density available for stable ion acceleration narrows with laser intensity decreasing. This problem at low laser intensities also requires very steep laser fronts such that the rise time of laser intensity is short compared to time in which these instabilities grow. For example, we have repeated our reference 3D simulation with same input parameters, but for a slowly rising pulse front, using a Gaussian $(l=1)$ rather than the super-Gaussian $(l=15)$. No IWBA was observed in this case. Thus in order to get the IWBA result under the condition of lower incident laser intensity, one has to use the plasma density very close to the threshold and a very steep front of the laser pulse. Pulse front steepening has been observed for laser pulses propagating in near-critical plasma [22]; this may become a solution to achieve the required contrast ratios.

In conclusion, we have explored a regime of ion acceleration in a laser-driven double layer wakefield where ions are self-injected by ion wave breaking. It is found that, just beyond the ion wave breaking threshold, ion beams with superior properties are generated. In particular in 3D geometry, ultrashort self-injection occurs localized close to the laser axis. This leads to highly collimated ion pulses with peaked ion energy spectra. The present results are important for near future experiments at laser intensities of $10^{21}-10^{23} \mathrm{~W} / \mathrm{cm}^{2}$, now becoming available.

B. Liu acknowledges support from the Alexander von Humboldt Foundation and from the National Natural Science Foundation of China (Grant No. 11205010). B. Liu, H. Ruhl, and K.-U. Bamberg acknowledge support by the Gauss Centre for Supercomputing(GCS) Large-Scale Project (Project No. pr92na), and the Cluster-of-Excellence Munich Centre for Advanced Photonics (MAP). B. Liu also thanks Professor J. Schreiber for providing computing resources on the Hydra supercomputer at Max-PlanckInstitute für Quantenoptik. B. Liu and J. Meyer-ter-Vehn had fruitful discussions with Professor Z. M. Sheng.

[1] M. Borghesi, Nuclear instruments and methods in physics research section A: accelerators, spectrometers, detectors and associated equipment, Nucl. Instrum. Methods Phys. Res., Sect. A 740, 6 (2014).

[2] S. V. Bulanov and V.S. Khoroshkov, Feasibility of using laser ion accelerators in proton therapy, Plasma Phys. Rep. 28, 453 (2002).

[3] V. Malka, J. Faure, Y. A. Gauduel, E. Lefebvre, A. Rousse, and K. T. Phuoc, Principles and applications of compact laser-plasma accelerators, Nat. Phys. 4, 447 (2008).

[4] D. Schardt, T. Elsässer, and D. Schulz-Ertner, Heavy-ion tumor therapy: Physical and radiobiological benefits, Rev. Mod. Phys. 82, 383 (2010).

[5] T. Tajima and J. M. Dawson, Laser electron accelerator, Phys. Rev. Lett. 43, 267 (1979).

[6] E. Esarey, C. B. Schroeder, and W. P. Leemans, Physics of laser-driven plasma-based electron accelerators, Rev. Mod. Phys. 81, 1229 (2009).

[7] A. Pukhov and J. Meyer-ter-Vehn, Laser wake field acceleration: the highly non-linear broken-wave regime, Appl. Phys. B: Lasers Opt. 74, 355 (2002).

[8] S. C. Wilks, W. L. Kruer, M. Tabak, and A. B. Langdon, Absorption of ultra-intense laser pulses, Phys. Rev. Lett. 69, 1383 (1992).

[9] A. Macchi, F. Cattani, T. V. Liseykina, and F. Cornolti, Laser acceleration of ion bunches at the front surface of overdense plasmas, Phys. Rev. Lett. 94, 165003 (2005).

[10] N. Naumova, T. Schlegel, V. T. Tikhonchuk, C. Labaune, I. V. Sokolov, and G. Mourou, Hole boring in a DT pellet and fast-ion ignition with ultraintense laser pulses, Phys. Rev. Lett. 102, 025002 (2009).

[11] C. A. J. Palmer, N. P. Dover, I. Pogorelsky, M. Babzien, G. I. Dudnikova, M. Ispiriyan, M. N. Polyanskiy, J. Schreiber, P. Shkolnikov, V. Yakimenko, and Z. Najmudin, Monoenergetic proton beams accelerated by a radiation pressure driven shock, Phys. Rev. Lett. 106, 014801 (2011). 
[12] X. Q. Yan, C. Lin, Z. M. Sheng, Z. Y. Guo, B. C. Liu, Y. R. Lu, J.X. Fang, and J. E. Chen, Generating high-current monoenergetic proton beams by a circularly polarized laser pulse in the phase-stable acceleration regime, Phys. Rev. Lett. 100, 135003 (2008).

[13] B. Qiao, M. Zepf, M. Borghesi, and M. Geissler, Stable GeV ion-beam acceleration from thin foils by circularly polarized laser pulses, Phys. Rev. Lett. 102, 145002 (2009).

[14] A. Macchi, S. Veghini, T. V. Liseykina, and F. Pegoraro, Radiation pressure acceleration of ultrathin foils, New J. Phys. 12, 045013 (2010).

[15] C. A. J. Palmer et al., Rayleigh-Taylor instability of an ultrathin foil accelerated by the radiation pressure of an intense laser, Phys. Rev. Lett. 108, 225002 (2012).

[16] O. Shorokhov and A. Pukhov, Ion acceleration in overdense plasma by short laser pulse, Laser Part. Beams 22, 175 (2004).

[17] T. Esirkepov, S. V. Bulanov, M. Yamagiwa, and T. Tajima, Electron, positron, and photon wakefield acceleration: trapping, wake overtaking, and ponderomotive acceleration, Phys. Rev. Lett. 96, 014803 (2006).
[18] B. Shen, Y. Li, M. Y. Yu, and J. Cary, Bubble regime for ion acceleration in a laser-driven plasma, Phys. Rev. E 76, 055402 (2007).

[19] H. Ruhl, Classical particle simulations, in Introduction to Computational Methods in Many Particle Body Physics, edited by M. Bonitz, and D. Semkat (Rinton, Paramus, New Jersey, 2006).

[20] S. M. Weng, M. Murakami, P. Mulser, and Z. M. Sheng, Ultra-intense laser pulse propagation in plasmas: from classic hole-boring to incomplete hole-boring with relativistic transparency, New J. Phys. 14, 063026 (2012).

[21] A. P. L. Robinson, P. Gibbon, M. Zepf, S. Kar, R. G. Evans, and C. Bellei, Relativistically correct hole-boring and ion acceleration by circularly polarized laser pulses, Plasma Phys. Controlled Fusion 51, 024004 (2009).

[22] J. H. Bin, W. J. Ma, H. Y. Wang, M. J. V. Streeter, C. Kreuzer, D. Kiefer, M. Yeung, S. Cousens, P. S. Foster, B. Dromey, X. Q. Yan, R. Ramis, J. Meyer-ter-Vehn, M. Zepf, and J. Schreiber, Ion acceleration using relativistic pulse shaping in near-critical-density plasmas, Phys. Rev. Lett. 115, 064801 (2015). 